\title{
F. Carrera Ramirez, et r. Fabregas Valcace, (dir.),Arte parietal megalitico en el Noroeste peninsular. Conocimiento y conservacion
}

\section{Charles-Tanguy Le Roux}

\section{(2) OpenEdition Journals}

Édition électronique

URL : http://journals.openedition.org/rao/525

DOI : $10.4000 /$ rao. 525

ISBN : 978-2-7535-1606-9

ISSN : $1775-3732$

Éditeur

Presses universitaires de Rennes

\section{Édition imprimée}

Date de publication : 30 décembre 2006

Pagination : 231-232

ISBN : 978-2-7535-0574-2

ISSN : 0767-709X

\section{Référence électronique}

Charles-Tanguy Le Roux, « F. Carrera Ramirez, et r. Fabregas Valcace, (dir.),Arte parietal megalitico en el Noroeste peninsular. Conocimiento y conservacion », Revue archéologique de l'Ouest [En ligne], 23 | 2006, mis en ligne le 30 décembre 2008, consulté le 25 décembre 2020. URL : http:// journals.openedition.org/rao/525 ; DOI : https://doi.org/10.4000/rao.525 
par rapport à celles de la Région de Castilla-la Mancha n’est pas toujours évident pour un lecteur étranger.

Tout ce travail s'appuie sur une série d'études techniques, certaines classiques d'autres plus pointues. Elles sont regroupées dans les ch. IX (pour le paléoenvironnement et l'économie) et $\mathrm{X}$ (pour ce qui concerne les hommes euxmêmes). L'ouvrage comporte une bibliographie de quelque 280 titres (plus celles des " annexes ») et son illustration est de qualité (avec un nombre non négligeable de documents en couleurs). Un petit regret : que le texte ne soit pas mieux structuré (avec davantage de sous-titres et de résumés), ce qui en aurait facilité la consultation pour les lecteurs non hispanophones de naissance : on y "rame " parfois, mais il est vrai que l'intérêt de l'ouvrage pour tout chercheur s'intéressant au mégalithisme mérite bien cet effort.

Charles-Tanguy Le Roux

Carrera Ramirez, F. et Fabregas Valcace, R. (dir.), 2006 - Arte parietal megalitico en el Noroeste peninsular. Conocimiento y conservacion, Santiago de Compostela, Torculo Edicions, 292 p. (ISBN 848408388 8).

Ce livre présente le bilan d'un projet de recherche soutenu par le Ministère espagnol de la Science et de la Technologie (au titre du FEDER) et par le Conseil régional de Galice. Après une présentation liminaire, l'ouvrage s'articule en sept chapitres, chacun étant précédé d'un résumé en anglais (parfois un peu succinct) et suivi de sa propre bibliographie.

Au chapitre 1, R. Fabregas et I. Vilaseco présentent les mégalithes galiciens dans leur contexte - humain essentiellement, l'environnement naturel n'étant quasiment pas abordé à ce stade - et dans leur fonctionnalité; on y trouve un très utile tableau récapitulatif des dates radiocarbone aujourd'hui disponibles - 67 dates pour quelque 30 monuments - (on pourrait discuter de la pertinence des « moyennes pondérées » introduites pour les sites ayant fourni plusieurs dates, mais les données princeps y sont et c'est là l'essentiel). Toujours en matière de chronologie, un graphique confronte les plus significatives de ces dates aux types architecturaux concernés, faisant ressortir une concentration des monuments ornés sur le début du $\mathrm{IV}^{\mathrm{e}}$ millénaire av. J.-C., malgré quelques sites apparemment pionniers (comme coto dos Mouros, dont le cas est d'ailleurs discuté plus loin).

Le chapitre 2 ( F. Carrera et R. Fabregas) présente les datations directes de peintures obtenues par AMS grâce à une collaboration avec l'université du Texas (9 dates sur 7 monuments, à partir de 12 prélèvements : les échantillons de l'emblématique dolmen de Dombate se sont hélas avérés inexploitables). Après un exposé méthodologique, la discussion des résultats fait ressortir là aussi une concentration sur la première moitié du IV $\mathrm{V}^{\mathrm{e}}$ millénaire avant J.-C., mais elle soulève trois problèmes rémanents : les reprises du décor (le meilleur cas est celui de Monte dos Marxos où deux phases ont été clairement distinguées sur la même dalle peinte), le broyage (fortuit ou délibéré) de charbon ancien pour la confection du pigment noir et le décor a posteriori d'un monument préexistant.
Au chapitre 3, F. Carrera passe en revue les techniques décoratives utilisées, dont la variété peut correspondre à des différences d'ordre technique (grain, dureté, état de surface de la roche support), mais aussi d'ordre culturel. Les " gravures " s.l. comprennent en fait des piquetages, des abrasions, des incisions et des reliefs en épargne tandis que les " peintures " s'avèrent appliquées soit directement soit sur un apprêt blanc, les deux techniques pouvant d'ailleurs être associées. Composition et préparation de cet apprêt sont bien entendu étudiées, ainsi que celles des pigments (blanc, noir et rouge). Pour les noirs, deux sources sont possibles, le carbone et le manganèse. Pour les rouges et ocres, le travail passe en revue les différents oxydes et hydroxydes de fer disponibles et discute de leur éventuelle calcination. La question du cinabre (le vermillon des peintres) est également abordée; bien plus difficile à obtenir, ce pigment est apparemment cantonné à des usages à forte connotation symbolique (comme le saupoudrage des squelettes d'Alberite). En ce qui concerne le liant (il est rappelé que, dès 1934, G. Leisner envisageait l'utilisation du blanc d'œuf), l'auteur relativise les affirmations de naguère sur l'utilisation de beurre de vache à Dombate; analyses à l'appui, il semble difficile de reconnaître plus que l'utilisation d'une "graisse animale ", ce qui n'est déjà pas si mal.

Ce chapitre est directement complété par le ch. 6, consacré (par F. Carrera, J. Suriol et B. Silva) à l'étude des matières picturales et des techniques par les méthodes modernes (microscopies optique et électronique, spectroscopie IR, diffraction X, porosimétrie, absorption capillaire), tant sur les pigments, liants et apprêts que sur les encroûtements. Sont également étudiés les processus de vieillissement, notamment à Dombate où se posent, comme on sait, de graves problèmes en ce domaine. Les expérimentations conduisent à des propositions en matière de conservation (cf. infra, ch. 7).

Ces chapitres passionneront tous ceux qui s'étonnent du développement de l'art pariétal peint dans le nord-ouest ibérique et de son absence dans les autres grands foyers d'art 
mégalithique (à commencer par l'Irlande et la Bretagne). Une telle disparité est étonnante quand on connaît le goût inné pour la couleur du cerveau humain, desservi par un œil au sens chromatique très développé. On a pu penser à des conditions de conservation particulièrement favorables en Ibérie (mais, pour quiconque a vu les monuments concernés, la différence semble pas flagrante); on a pu aussi envisager des techniques picturales spécialement élaborées (mais toutes les peintures qui nous sont parvenues ne bénéficient pas d'une couche d'apprêt élaborée et les rares traces de " fusain » conservées en région parisienne montrent que, même appliqués à sec, les pigments peuvent subsister). On peut aussi penser à une palette différente, recourant à des teintures organiques : à ce propos, qui sait si les compositions ibériques n'étaient pas initialement complétées, par exemple avec des verts (la couleur fétiche des parures en pierres fines...), des bleus ou des violets aujourd'hui totalement évanouis? Le débat - fondamental pour approcher la sensibilité des hommes néolithiques - reste ouvert.

Au chapitre 4, P. Bueno et R. de Balbin élargissent le sujet à l'ensemble de la péninsule ibérique, dans le cadre de la façade atlantique de l'Europe. Leur "état de la question " montre l'immense chemin parcouru depuis la synthèse pionnière d'E. Shee (1981) et le débat qui s'ensuit est trop riche pour pouvoir être résumé en quelques lignes. On en retiendra les rapports envisagés entre art mobilier (les plaquettes) et art pariétal, entre stèles, idoles et orthostates ornés, entre art ostensible et art caché, entre thématiques régionales et logiques territoriales. Puis les auteurs envisagent pour cet art mégalithique des racines remontant au fonds culturel des premiers paysans européens, voire à un tréfonds hérité des derniers chasseurs - cueilleurs et valorisant des ancêtres mythifiés. Enfin, un lien est proposé avec l'émergence des chefferies de l'âge du Bronze.

Avec le chapitre 5, T. Rivas, B. Prieto et B. Silva nous ramènent en Galice pour étudier les matériaux de construction des mégalithes par rapport aux ressources du substrat géologique régional (à la différence de la Bretagne, les cas de transport à longue distance - plus de $1 \mathrm{~km}$ - semblent ici rarissimes), à la topographie et aux caractéristiques mécaniques des roches (selon sa résistance à la flexion et à la compression, suivant la foliation ou perpendiculairement à celle-ci, tel ou tel matériau s'avère plus apte à la réalisation d'orthostates ou de tables de couverture). Sont également abordés différents aspects de ce que D. Sellier (1991; 1995) avait appelé la "météorisation postmégalithique ». Par contre, les méthodes d'appropriation des matériaux ne sont pratiquement pas abordées, peut-être parce que le relief relativement accidenté de Galice, en multipliant les bons affleurements de roches à débitage tabulaire, rendait inutile le recours à des techniques d'extraction susceptibles de laisser des stigmates significatifs, comparables à ceux relevés par D. Sellier (op. cit.) puis par E. Mens (2002) dans le Morbihan, ou tout récemment par M. Le Goffic (2006, p. 68-99) en Finistère.

Au chapitre 7 enfin, F. Carrera tire les conclusions de toutes ces études pour proposer des "aides à la décision" à l'usage des experts et gestionnaires en charge de l'exceptionnel patrimoine mégalithique galicien, confronté aux outrages de la nature et surtout des hommes: les seuls facteurs anthropiques d'altération sont estimés à $60 \% \mathrm{du}$ risque total (et à plus de $95 \%$ pour ce qui concerne l'environnement!). Face à une situation préoccupante (et encore a-t-elle été évaluée avant les dramatiques incendies de l'été dernier), des solutions sont proposées. Elles sont drastiques mais réalistes : le remblaiement est demandé pour 26 des 45 monuments passés en revue, des aménagements lourds sont proposés pour trois autres et le statu-quo est accepté pour 19 ... Reste à espérer que les actes suivront quand on connaît le sort réservé depuis des années au malheureux dolmen de Dombate, tout emblématique qu'il soit.

En résumé, un ouvrage très riche aussi bien pour les chercheurs que pour les gestionnaires du patrimoine et, ce qui ne gâte rien, d'une présentation fort agréable. À lire ou à se faire traduire - absolument.

Charles-Tanguy Le Roux

LE Goffic, M., 2006 - La nécropole mégalithiquede la pointe du Souch en Ploubinec (Finistère), (document final de synthèse de fouille triennale), Le Faou, Conseil général du Finistère, service départemental d'Archéologie, $147 \mathrm{p}$.

Mens, E., 2002 - L'affleurement partagé. Gestion du matériau mégalithique et chronologie de ses représentations gravées dans le Néolithique moyen armoricain, (Thèse de doctorat), université de Nantes.

SELLIER, D., 1991 - Analyse morphologique des marques de la météorisation des granites à partir de mégalithes morbihannais - l'exemple de l'alignement de Kerlescan à Carnac, Revue archéologique de l'Ouest, 8, p. 83-97.

SELLIER, D., 1995 - Éléments de reconstitution du paysage prémégalithique sur le site des alignements de Kerlescan (Carnac, Morbihan), à partir de critères géomorphologiques, Revue archéologique de l'Ouest, 12, p. 21-41. 